\title{
MicroRNA-29b targeting of cell division cycle 7-related protein kinase (CDC7) regulated vascular smooth muscle cell (VSMC) proliferation and migration
}

\author{
Qunhua Ma ${ }^{1 \#}$, Jing Zhang" ${ }^{2 \#}$ Ming Zhang ${ }^{3}$, Huan Lan ${ }^{4}$, Qian Yang ${ }^{5}$, Chengping Li ${ }^{2}$, Li Zeng ${ }^{6}$ \\ ${ }^{1}$ RICU\&MICU, Sichuan Provincial People's Hospital, University of Electronic Science and Technology of China, Chengdu, China; ${ }^{2}$ Emergency \\ Observation Ward, Sichuan Provincial People's Hospital, University of Electronic Science and Technology of China, Chengdu, China; ${ }^{3}$ Cancer \\ Center, Sichuan Provincial People's Hospital, University of Electronic Science and Technology of China, Chengdu, China; ${ }^{4}$ Department of \\ Cardiovascular Medicine, Southwest Medical University, Luzhou, China; ${ }^{5}$ School of Nursing, Chengdu Medical College, Chengdu, China; \\ ${ }^{6}$ Department of Nursing, Sichuan Provincial People's Hospital, University of Electronic Science and Technology of China, Chengdu, China \\ Contributions: (I) Conception and Design: C Li, L Zeng; (II) Administrative support: Q Ma, J Zhang; (III) Provision of study materials or patients: \\ C Li, L Zeng; (IV) Collection and assembly of data: M Zhang, H Lan; (V) Data analysis and interpretation: Q Yang; (VI) Manuscript writing: All \\ authors; (VII) Final approval of manuscript: All authors. \\ "These authors contributed equally to this work. \\ Correspondence to: Chengping Li. Emergency Observation Ward, Sichuan Provincial People's Hospital, University of Electronic Science and \\ Technology of China, Chengdu 611731, China. Email: lcp87393555@sina.com; Li Zeng. Department of Nursing, Sichuan Provincial People’s \\ Hospital, University of Electronic Science and Technology of China, Chengdu 611731, China. Email: 2174221755@qq.com.
}

Background: Proliferation and migration of vascular smooth muscle cells (VSMCs) are vital processes in vascular remodeling and pathology. This study aimed to explore the expression of miR-29b and cell division cycle 7-related protein kinase (CDC7) in patients with cerebral aneurysm (CA) and their effects on the proliferation and mobility of human umbilical artery smooth muscle cells (HUASMCs).

Methods: RNA levels of miR-29b and CDC7 were evaluated in the CA tissues and adjacent normal cerebral arteries from 18 patients undergoing surgery for CA rupture. The targeting of CDC7 by miR-29b was verified with luciferase reporter assay. Both CDC7 and miR-29b overexpression and silencing vectors were introduced to validate their effects on the proliferation and mobility of HUASMCs.

Results: The mRNA level of miR-29b was down-regulated $(\mathrm{P}<0.05)$, while the mRNA level of CDC7 was markedly elevated in CA patients $(\mathrm{P}<0.05)$. A Luciferase reporter assay showed CDC7 is a target gene of miR-29b, and miR-29b mimic down-regulated the mRNA and protein levels of CDC7 $(\mathrm{P}<0.05)$. Furthermore, miR-29b mimic inhibited, while miR-29b inhibitor or CDC7 over-expression promoted the proliferation and mobility of HUASMCs $(\mathrm{P}<0.05)$.

Conclusions: miR-29-3p inhibits cell proliferation and mobility via directly targeting CDC7, which could be a potential therapeutic target for vascular dysfunction related diseases, including atherosclerosis and CA.

Keywords: Atherosclerosis; cerebral aneurysm (CA); cell division cycle 7-related protein kinase (CDC7); miR29b-3p; intracranial aneurysm (IA); proliferation; metastasis

Submitted Aug 28, 2020. Accepted for publication Nov 06, 2020.

doi: $10.21037 / \mathrm{atm}-20-6856$

View this article at: http://dx.doi.org/10.21037/atm-20-6856 


\section{Introduction}

Phenotypic switching of the vascular smooth muscle cell (VSMC) is the initial event of a cerebral aneurysm (CA) and atherosclerosis (1), both of which are common vascular diseases with high morbidity and mortality $(2,3)$. The pathogenesis of CA and atherosclerosis are still mostly unclear. Current related factors focused on acquiring hemodynamic changes in artery walls, genetic factors, and pathological changes to blood vessels (4). Smooth muscle cells in vessels are critical to vascular remodeling and formation of an aneurysm. The proliferation and movement of VSMCs are related to vascular balance and stability (5). Thus, inhibiting the proliferation and movement of VSMCs may contribute to CA and atherosclerosis therapy.

MicroRNA-29b (miR-29b) was found to be downregulated in CA (6). The knockdown of miR-29b significantly promoted proliferation, migration, and phenotypic switching of VSMCs (7). Moreover, miR-29b is elevated in the serum of atherosclerotic patients and may affect the migration and proliferation of VSMCs $(8,9)$. These showed miR-29b might be correlated with CA and atherosclerosis, and supplement of the miR-29b level might be a potential strategy for CA and atherosclerosis.

Accumulating evidence indicated that cell division cycle 7-related protein kinase (CDC7), an essential part of eukaryotic DNA replication initiation, played a fundamental role in tumorigenesis and represented a novel promising therapeutic target of cancer $(10,11)$. It is not clear whether CDC7 participates in DNA replication, initiation of VSMCs, nor understanding of its role in CA or atherosclerosis. Similarities are usually observed in the pathogenesis of CA, atherosclerosis, and some tumors, especially in proliferation, differentiation, and inflammation, which are primarily involved in CA and atherosclerosis progression (1). More importantly, CDC7 was critical of some gene expression in smooth muscle cells, especially phenotypic maturation (12). CDC7 may take part in VSMC proliferation in CA and atherosclerosis.

In the present study, we aim to clarify whether miR29b and CDC7 could be associated with CA. The effects of miR-29b and CDC7 on VSMCs were shown by the proliferation and mobility of human umbilical artery smooth muscle cells (HUASMCs).

We present the following article in accordance with the MDAR reporting checklist (available at http://dx.doi. org/10.21037/atm-20-6856).

\section{Methods}

\section{Clinical samples}

CA tissues and adjacent normal cerebral arteries were collected from 18 patients ( 8 women and 10 men, aged 52-77, ethnic Han) during surgery for CA rupture at Sichuan Provincial People's Hospital as perversely described (13). CA was diagnosed according to digital subtraction angiography. Written informed consent was obtained from all CA patients. The Research Ethics Committee approved the study of the Sichuan Provincial People's Hospital (approval No. 2018-51), according to the Declaration of Helsinki (as revised in 2013) and Title 45, US Code of Federal Regulations.

\section{Cell culture and viability}

HUASMCs were bought from ATCC and kept in the Dulbecco's Modified Eagle Medium (DMEM, Gibco, USA) supplement by $10 \%$ fetal bovine serum (FBS, Gibco), $50 \mathrm{U} / \mathrm{mL}$ penicillin G (Gibco), and $250 \mu \mathrm{g} / \mathrm{mL}$ streptomycin (Gibco) at $37^{\circ} \mathrm{C}$ in a humidified incubator of $5 \% \mathrm{CO}_{2}$. For assessment of cell viability, 3,000 transfected cells were seeded into each well of a 96-well plate with $100 \mu \mathrm{L}$ medium, incubated for 22 hours before $10 \mu \mathrm{L}$ of CCK-8 was added. A microplate reader evaluated the optical densities of wells after incubating for more 2 hours.

\section{Quantitative real-time polymerase chain reaction (qRT-PCR)}

Total RNA was extracted from tissues and HUASMCs using TRIzol reagent (Invitrogen, Carlsbad, USA) according to the manufacturer's protocol. Briefly, eye scissors carefully separated CA tissues, ground in liquid nitrogen, and $1 \mathrm{~mL}$ of TRIzol was added to each sample, vortexed and centrifuged at $12,000 \times \mathrm{g}, 4^{\circ} \mathrm{C}$ for 10 minutes. The supernate was then collected and kept at room temperature for 5 minutes before chloroform $(0.2 \mathrm{~mL})$ was added, vortexed for 15 seconds, and kept at room temperature for another 2 minutes. After centrifuging at $12,000 \times \mathrm{g}, 4^{\circ} \mathrm{C}$ for 15 minutes, the aqueous phase was added with $0.5 \mathrm{~mL}$ isopropanol overnight to harvest total RNA. Concentrations of total RNA were evaluated and adjusted with RNasefree water. The first-strand cDNA was transcribed with the extracted RNA and SuperScript Reverse Transcriptase Kit (Vazyme, Nanjing, China). An SYBR Green PCR Master 
Table 1 Primers used in PCR

\begin{tabular}{lll}
\hline Gene name & \multicolumn{1}{c}{ Forward 5'-3' } & Reverse 5'-3' \\
\hline CDC7 & AGTGCCTAACAGTGGCTGG & CACGGTGAACAATACCAAACTGA \\
miR-29b-3p & TGCGGTAGCACCATTTGAAAT & CCAGTGCAGGGTCCGAGGT \\
snRNA U6 & CTCGCTTCGGCAGCACA & AACGCTTCACGAATTTGCGT \\
GAPDH & CCAGGTGGTCTCCTCTGACTTC & GTGGTCGTTGAGGGCAATG \\
\hline
\end{tabular}

PCR, polymerase chain reaction; CDC7, cell division cycle 7-related protein kinase.

Mix (Vazyme) kit was employed to show the relative mRNA levels of miR-29b and CDC7in, a fast-real-time PCR 7300 System (Applied Biosystems, Foster City, USA). Primers for miR-29b, CDC7, U6, and GAPDH were all synthesized by Sangon Biotech (Shanghai, China) (See Table 1). Data were analyzed according to the comparative Ct. Levels of miR-29b and CDC7 were normalized to U6 and GAPDH, respectively.

\section{Cell transfection}

siRNA-CDC7, miR-29b mimic, and miR-29b inhibitor were designed and synthesized by Ribobio (Guangzhou, China). $\mathrm{pcDNA}^{\mathrm{TM}} 3.1$ was purchased from Invitrogen (USA). A total of $1 \times 10^{4}$ HUASMCs in $1 \mathrm{~mL}$ medium was seeded into each well of a six-well plate. After incubation for 24 hours, $50 \mathrm{nM}$ of pcDNA ${ }^{\mathrm{TM}} 3.1-\mathrm{CDC} 7$ (pc-CDC7), miR-29b mimic, miR-29b inhibitor, or siRNA-CDC7 was transfected into each well using Lipofectamine ${ }^{\circledR} 3000$ (Invitrogen) respectively or in combination according to the group. A scrambled miRNA (scramble) was used as control.

\section{Western blot}

HUASMCs were lysed with RIPA Lysis Buffer (Beyotime Institute of Biotechnology). Thirty micrograms of protein for each sample in the loading buffer were separated by $10 \%$ SDS-PAGE gel. After transferring to a PVDF membrane (Millipore, Billerica, MA, USA) and blocked with 5\% skim milk in TBST, primary antibodies (Abcam, Cambridge, MA, USA) and HRP-conjugated secondary antibody (Abcam, ab6721) were incubated. Finally, the blots were visualized by ECL and detected using a ChemiDoc XRS imaging system. Primary antibodies used were: GAPDH (ab9482, 1:3,000, $37 \mathrm{kDa}$ ), Ki67 (ab16667,1:1,000, $359 \mathrm{kDa}$ ), PCNA (ab92552, 1:2,000, $29 \mathrm{kDa}$ ), matrix metalloprotein-2 (MMP-2) (ab37150, 1:2,000, $72 \mathrm{kDa}$ ), alpha-smooth muscle actin ( $\alpha$-SMA) (ab7817, 1:2.94, $40 \mathrm{kDa}$ ), smooth muscle
Myosin heavy chain 11 (SM-MHC) (ab133567, 1:2,000, $200 \mathrm{kDa}$ ) and CDC7 (ab229187, 1:1,000, $64 \mathrm{kDa})$.

\section{Luciferase reporter assay}

The targeting of CDC7 3'-UTR by miR-29b was predicted with Targetscan 7.2. The 3'-UTR of CDC7 mRNA containing predicted GenePharma synthesized miR-29b target sites or mutant binding sites according to previous research (14). Then, the synthesized RNA was PCRamplified and inserted into the pmirGLO dual-luciferase expression vector (Promega, Madison, WI, USA). For luciferase reporter assay, the inserted vectors for wildtype and mutant CDC7 3'-UTR, scrambled miRNA mimics, and miR-29b mimic was transfected into HEK $293 \mathrm{~T}$ by Lipofectamine 3000 regent. The luciferase activities were measured with a dual-luciferase reporter assay system (Promega) according to the manufacturer's instructions 48 hours later. Data were normalized to renilla activity.

\section{Transwell assay}

HUASMCs transfected with pc-CDC7, or miR-29b inhibitor or mimic, was cultured in a 24-well chamber. Transwell was performed to show the mobility of HUASMCs. Briefly, HUASMCs transfected with miR-29b inhibitor, or mimic or pc-CDC7, were seeded in the upper chamber in DMEM supplemented with $0.1 \%$ FBS, and the lower chamber was added with DMEM supplemented with $10 \%$ FBS. After incubating for 24 hours, the bottom HUASMCs were fixed in $95 \%$ ethanol, stained with hematoxylin, and observed with a DM2500 bright field microscope. The number of invaded HUASMCs was counted on 10 random fields in each well.

\section{Wound healing assay}

A total of $2 \times 10^{5}$ HUASMCs were seeded in each well of a 

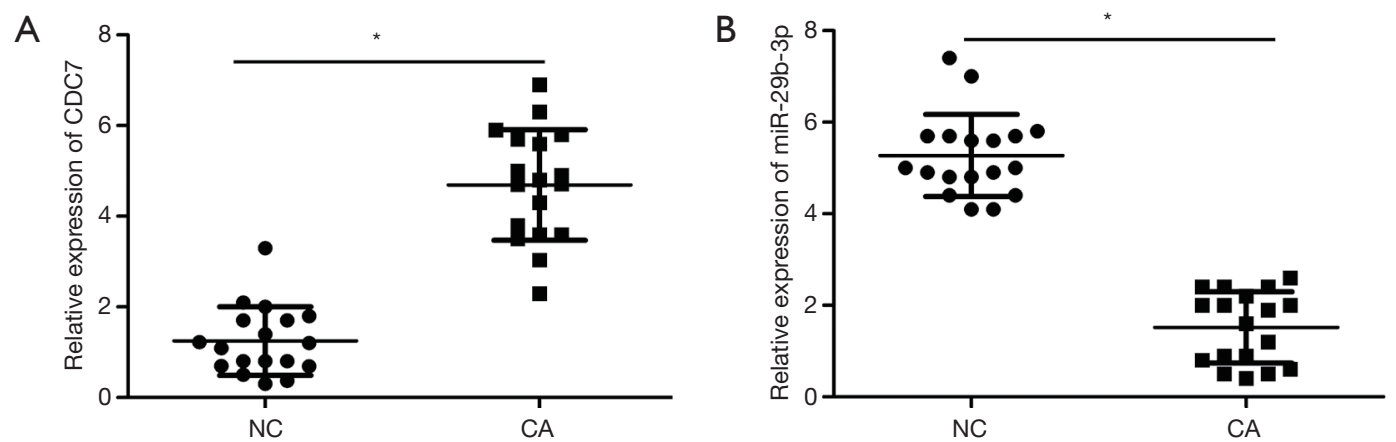

Figure 1 Elevated mRNA expression of CDC7 (A) and depressed miR-29b (B) in CA focus compared to adjacent tissues. Samples were from 18 patients undergoing surgery for CA rupture, 14 were women, and 4 were men, aged 40-65. NC, adjacent normal vessels; CA, cerebral aneurysm; CDC7, cell division cycle 7-related protein kinase; miR-29b, microRNA-29b. *, $\mathrm{P}<0.05$ versus NC.

6-well plate. Until the cells reached a confluence of 90-95\%, wound gaps were carefully created with a sterile $200 \mu \mathrm{L}$ pipette tip. The HUASMCs were washed twice with PBS to remove debris. A proliferation inhibitor mitomycin $\mathrm{C}$ $(10 \mu \mathrm{g} / \mathrm{mL})$ was added to the cell culture medium to inhibit cell replication (15). Then the HUASMCs were cultured in complete medium for 24 hours with or without mimic/ inhibitor. The same marked areas were re-photographed and calculated using Image J software. Images were acquired with a light microscope (Olympus, Tokyo, Japan) at 100x magnification.

\section{5-Ethynyl-2-Deoxyuridine (EdU) stains for cell proliferation}

HUASMCs in each group were treated and cultured in a 24-well chamber. Cells were incubated in $50 \mathrm{nmol} / \mathrm{L} \mathrm{EdU}$ solutions (RiboBio, Guangzhou, China) for 2 hours before visualizing. The EdU incorporation assay was applied following the manufacturer's instruction. Image J software counts EDU positive cell numbers.

\section{Bioinformatics}

The interaction between miR-29b(-3p) and CDC7 was predicted using TargetScan 7.2 (http://www.targetscan.org).

\section{Statistical analysis}

All quantitative data were presented as mean \pm standard deviation (SD) and analyzed using the SPSS software (SPSS Inc., Chicago, IL, USA, version 19.0). All experiments were independently technically repeated at least 3 times and data were analyzed using a one-way analysis of variance (ANOVA) test or student's $t$-test. Values of $\mathrm{P}<0.05$ were considered statistically significant.

\section{Results}

\section{miR-29b and $C D C 7$ are dysregulated in buman $C A$}

The mRNA levels of miR-29b and CDC7 in CA tissues and adjacent normal tissues from 18 patients were examined by qRT-PCR to understand the biological significance of miR$29 \mathrm{~b}$ and $\mathrm{CDC} 7$ in human CA. The expression of CDC7 in CA tissues was significantly higher than that in normal tissues (Figure $1 A, \mathrm{P}<0.05$ ), while $\mathrm{miR}-29 \mathrm{~b}$ was downregulated (Figure $1 B, \mathrm{P}<0.05$ ).

\section{miR-29b mimic or knockdown of CDC7 inbibits proliferation and migration of HUASMCs}

Since miR-29b and CDC7 are dysregulated in human CA, gain-of-function studies are performed in HUASMCs. miR-29b mimic, miR-29b inhibitor, siRNA-CDC-7, and scrambled miRNAs (scrambles) were transfected into HUASMCs, respectively. Consequently, the RNA expression of miR-29b was significantly elevated by mimics and reduced by inhibitors compared with the scramble (Figure $2 \mathrm{~A}$, $\mathrm{P}<0.01)$. Transfection of siRNA-CDC-7 significantly reduced mRNA expression of CDC-7 (Figure $2 B, \mathrm{P}<0.05$ ). The proliferation of HUASMCs indicated with relative expression pf Ki67 and PCNA were significantly inhibited with miR-29b mimic and siRNA-CDC-7 while promoted by a miR-29b inhibitor (Figure $2 C, D, \mathrm{P}<0.05$ ), which showed that siRNA-CDC-7 and miR-29b mimic significantly 
A

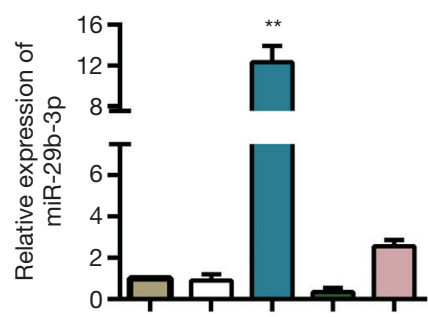

Scramble miR-29b mimic miR-29b inhibito

$\mathrm{D}$

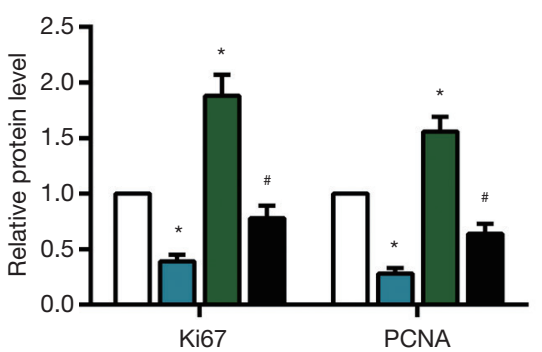

G

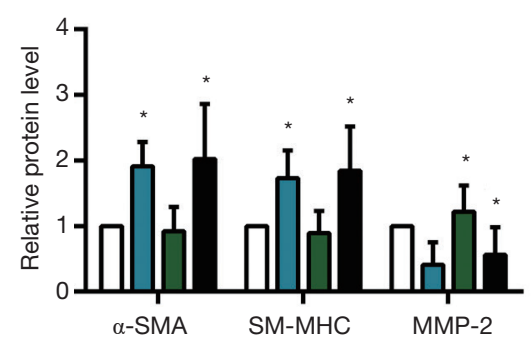

B

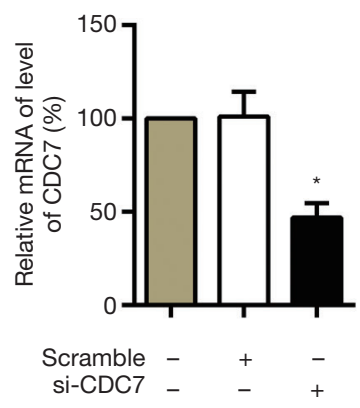

C

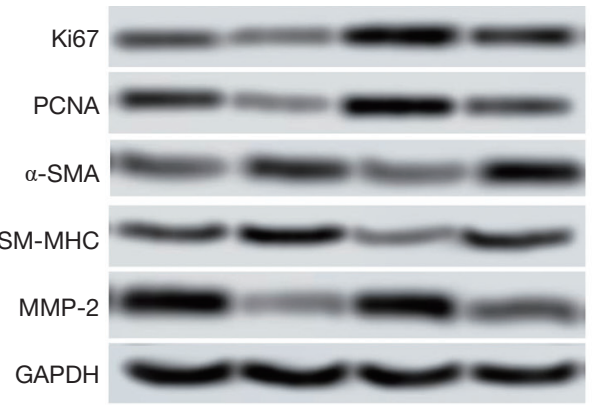

E
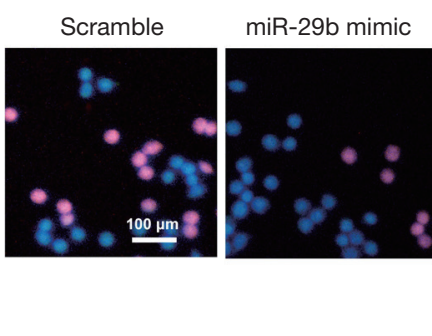

$\mathrm{H}$

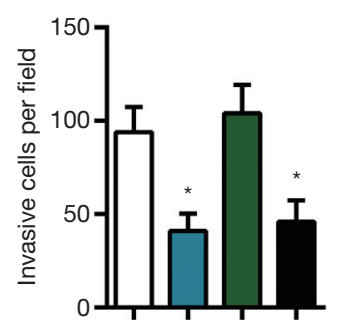

$\mathrm{F}$
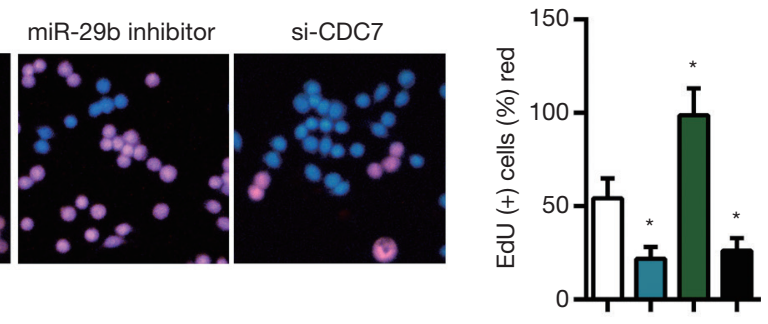

Figure 2 siRNA-CDC-7 and miR-29b mimic inhibit proliferation and migration of HUASMCs. HUASMCs were transfected with siRNA-CDC-7, miR-29b scramble, miR-29b mimic, or miR-29b inhibitor, then kept for another 24 hours for later experiments. (A) miR-29b mRNA expression in different groups determined by RT-PCR. **, $\mathrm{P}<0.01$, compared with the miR-29b scramble group. (B) Relative RNA expression of HUASMCs transfected with scramble and siRNA-CDC-7. *, P<0.05, compared with the miR-29b scramble group. (C) Relative protein levels of Ki67, PCNA, $\alpha$-SMA, SM-MHC, and MMP-2 were evaluated with western blotting. (D) Ki67 and PCNA levels were quantified with Image $\mathrm{J},{ }^{*}, \mathrm{P}<0.05$, compared with the miR-29b scramble group. ${ }^{\text {"}}, \mathrm{P}<0.05$, compared with the miR29b mimic group. (E) Cell proliferation was detected by EdU staining. Cells in red fluorescence represented the positive cell stained with EdU, and those in blue were stained with DAPI. (F) EdU positive cell count. *, $\mathrm{P}<0.05$, compared with the miR-29b scramble group. (G) Relative protein levels of $\alpha$-SMA, SM-MHC, and MMP-2 quantified with Image J. *, P<0.05, compared with the miR-29b scramble group. (H) Transwell tests detected cell migration, and (I) the wound healing of the transfected cells. MMP-2, matrix metalloprotein-2; a-SMA, alpha-smooth muscle actin; SM-MHC, smooth muscle Myosin heavy chain 11; miR-29b, microRNA-29b; HUASMCs, human umbilical artery smooth muscle cells; RT-PCR, real-time polymerase chain reaction; EdU, 5-Ethynyl-2-Deoxyuridine; DAPI, 4',6-diamidino-2-phenylindole.

inhibited the growth of HUASMCs, while miR-29b inhibitors promoted the proliferation. EDU positive cells in HUASMCs were significantly decreased with siRNACDC-7 and miR-29b mimic while increased with miR-29b inhibitor (Figure $2 E, F, \mathrm{P}<0.05)$. Also, markers of VSMC phenotypic switching were tested, $\alpha$-SMA, and SM-MHC was induced with miR-29b mimic or siRNA-CDC-7, while MMP-2 was inhibited (Figure $2 C, G, \mathrm{P}<0.05$ ). These 


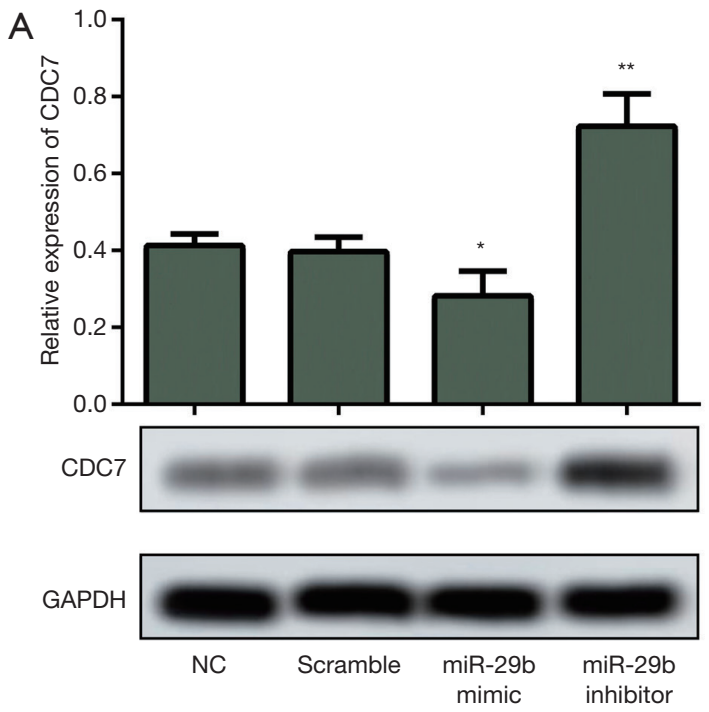
B Position 681-687 5' ...UCCUCGgAAAUUACAUGgugCug.. of CDC7 3' UTR | | | || ||
hsa-miR-29b 3' UUGUGACUAAAGUUUACCACGAU
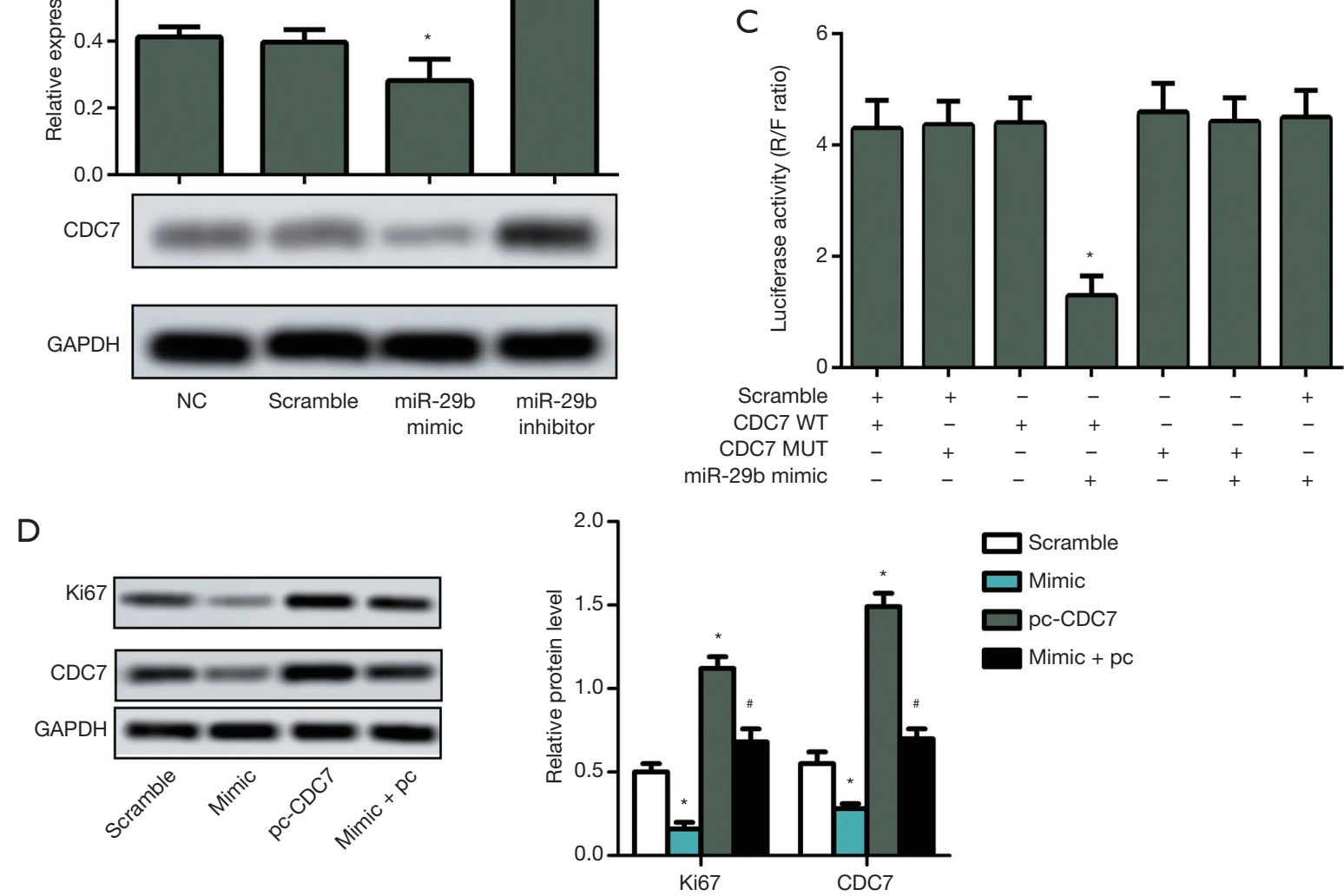

Figure 3 CDC7 was verified as a functional target of miR-29b. HUASMCs were transfected with miR-29b scramble, miR-29b mimic, or miR-29b inhibitor, then kept for another 24 hours for later experiments. (A) The protein expression of CDC7 was verified by western blot assay. *, $\mathrm{P}<0.05$ and ** $\mathrm{P}<0.01$ compared with the miR-29b scramble group. (B) TargetScan online prediction of combination between CDC7 and miR-29b. HEK 293T cells were co-transfected with CDC7 WT, CDC7 MUT, and miR-29b mimic, and (C) the target relationship between CDC7 and miR-29b was confirmed by Luciferase reporter assay. * $\mathrm{P}<0.05$, compared with the CDC7 WT group. HUASMCs transfected with miR-29b scramble, miR-29b mimic, or pc-CDC7. (D) The protein levels of CDC7 and Ki67 were verified by western blot assay. *, $\mathrm{P}<0.05$, compared with the scramble group; ${ }^{*}, \mathrm{P}<0.05$, compared with the pc-CDC7 group. CDC7, cell division cycle 7-related protein kinase; HUASMC, human umbilical artery smooth muscle cells; WT, wild-type; MUT, mutant; miR-29b, microRNA-29b.

findings implied that phenotypic switching was inhibited with miR-29b and promoted with CDC-7. Expectedly, miR-29b mimic, and siRNA-CDC-7 significantly decreased the invasive cells per field and wound closure rate of HUASMCs, while miR-29b inhibitors promoted the migration of HUASMCs (Figure $2 H, I, \mathrm{P}<0.05$ ).

\section{$C D C 7$ is verified as a functional target of $m i R-29 b$}

The protein expression of CDC7 was decreased by miR- 29b mimic but increased by miR-29b inhibitor, as shown in Figure $3 A(\mathrm{P}<0.05$ and $\mathrm{P}<0.01$ respectively). TargetScan online was applied to predict the targets of miR-29b. The results showed that $\mathrm{CDC} 7$ might be a potential target of miR-29b (Figure 3B). To verify this hypothesis, HUASMCs were co-transfected with the CDC7 reporter plasmid and miR-29b mimic. Therefore, the luciferase activity in miR-29b mimic and CDC7 wildtype co-transfected cells was significantly reduced compared to mutant $\mathrm{CDC} 7$ or scrambled RNA transfected cells (Figure $3 C, \mathrm{P}<0.05$ ). For 
A

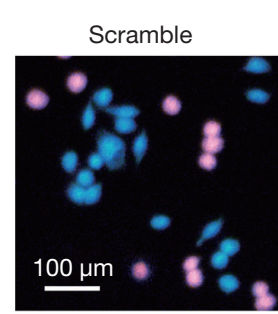

B

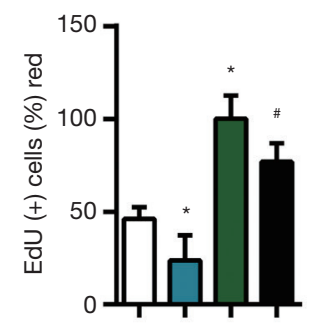

miR-29b mimic

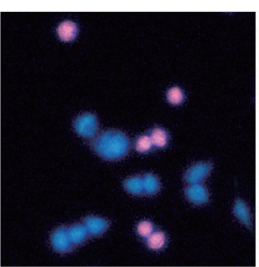

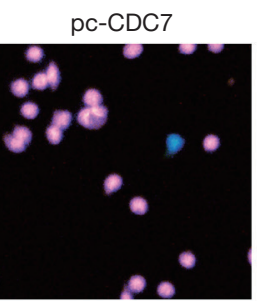

miR-29b mimic

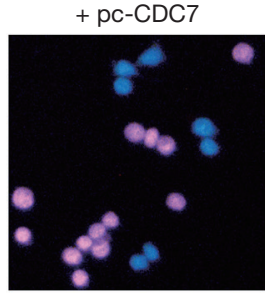

C

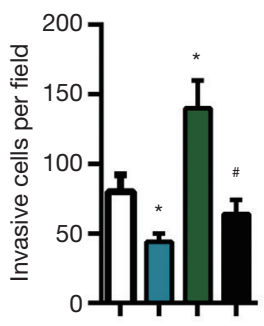

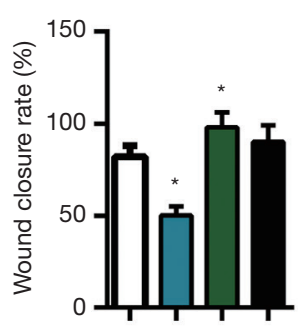

\section{E}
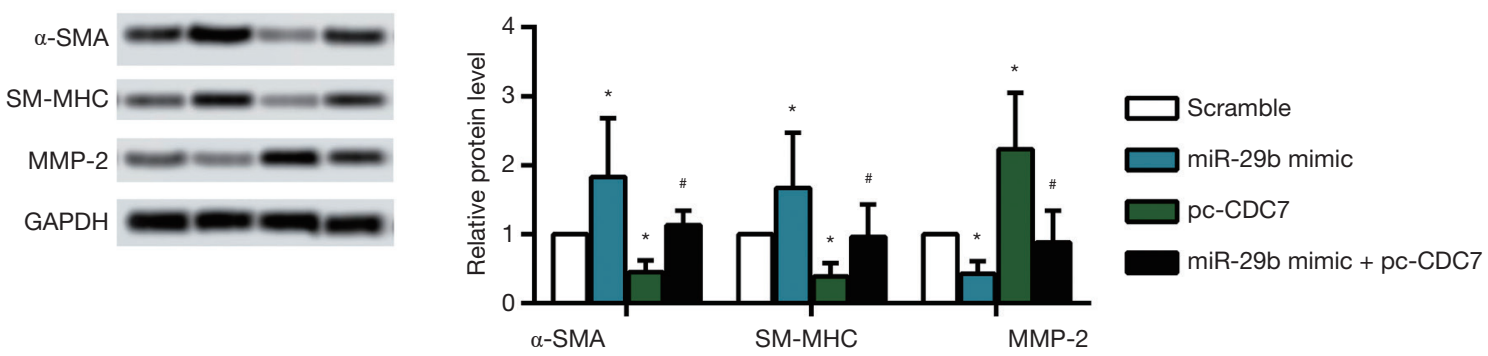

Figure 4 miR-29b targets CDC7 to inhibit proliferation and migration of HUASMCs. HUASMCs were transfected with miR-29b scramble, miR-29b mimic, or pc-CDC7, then kept for another 24 hours for later experiments. (A) Cell proliferation was detected by EdU staining. Cells in red fluorescence represented the positive cell stained with EdU, and those in blue were stained with DAPI. (B) EdU positive cell count. *, $\mathrm{P}<0.05$ compared with the scramble group; ${ }^{*}, \mathrm{P}<0.05$, compared with the pc-CDC7 group. (C) Cell mobility detected by Transwell assay, and (D) cell migration detected by wound healing assay. *, $\mathrm{P}<0.05$, compared with the scramble group; ${ }^{*}, \mathrm{P}<0.05$, compared with the pc-CDC7 group. (E) The protein levels of $\alpha$-SMA, SM-MHC, and MMP-2 were verified by western blot assay and quantified with Image J. *, $\mathrm{P}<0.05$, compared with the scramble group; ${ }^{*}, \mathrm{P}<0.05$, compared with the pc-CDC7 group. CDC7, cell division cycle 7-related protein kinase; miR-29b, microRNA-29b; HUASMCs, human umbilical artery smooth muscle cells; EdU, 5-Ethynyl-2Deoxyuridine; DAPI, 4',6-diamidino-2-phenylindole; $\alpha$-SMA, alpha-smooth muscle actin; SM-MHC, smooth muscle Myosin heavy chain 11; MMP-2, matrix metalloprotein-2.

CDC7 initiates NDA replication, inhibition of CDC7 arrests the cell cycle. Consistent with former studies, we showed inhibition of migration and proliferation with miR29b mimic, through significantly inhibited Ki67 and CDC7 (Figure $3 D, \mathrm{P}<0.05$ ).

\section{miR-29b targets CDC7 to inbibit proliferation and migration of HUASMCs}

As shown in Figure 4A,B, miR-29b mimic significantly decreased the EdU positive cells in HUASMCs $(\mathrm{P}<0.05)$, while pc-CDC7 promoted the proliferation $(\mathrm{P}<0.05)$. Besides, invasive cells per field were decreased with miR29b mimic while increased with pc-CDC7 (Figure 4C, $\mathrm{P}<0.05)$. miR-29b mimic significantly decreased the wound closure of HUASMCs, while pc-CDC7 promoted the mobility of HUASMCs (Figure $4 D, \mathrm{P}<0.05$ ). Expectedly, miR-29b mimic significantly inhibited phenotypic switching of HUASMCs, while pc-CDC7 promotes the phenotypic switching of HUASMCs (Figure $4 E, \mathrm{P}<0.05$ ). 


\section{Discussion}

CA, also named as intracranial aneurysm (IA), refers to pathological dilatation of the cerebral artery caused by weakness of a cerebral artery lumen or vein wall, is still poorly studied and treated (16). CA is the leading cause of subarachnoid hemorrhage. Reduction or dysplasia of VSMCs is responsible for remodeling and thinness of the artery wall, resulting in the development of CA (4). Moreover, down-regulation of miR-29b allows MMP2 expression and vascular calcification (17), and targets growth factors and cytoskeleton to regulate VSMC phenotypic switching and thus vascular elasticity (18), all of which potentially elevates blood pressure and promote $\mathrm{CA}$ progression. Here, we evaluate the role of miR-29b and CDC7 in CA and their effects on the proliferation and migration of HUASMCs.

Differently expressed miRNAs were found in CA tissues and serum of patients, including miR-29b as a vital member $(13,19)$. miR-29b was broadly reported to be related to cancer $(20,21)$, insulin resistance (22), and cardiocyte protection (23). Meanwhile, miR-29b was demonstrated to be involved in cell apoptosis, autophagy, proliferation, and migration $(24,25)$. For example, miR-29b inhibited the proliferation and promoted apoptosis in multiple myeloma (26); or suppressed proliferation and mobility of pancreatic cancer cells (27). Interestingly, miR-29b promoted cell apoptosis on smooth muscle cells $(18,28)$, suggesting that miR-29b may be promising in treating many proliferative vascular disorders, including CA. miR$29 \mathrm{~b}$ was demonstrated to be down-regulated in CA $(13,19)$. In the present study, our experiments verified miR-29b was indeed down-regulated in CA tissues. Also, increasing the expression of miR-29b by mimicking transfection inhibited the proliferation and migration of HUASMCs, while miR-29b inhibitors exhibited the opposite results, which indicated that low expression of miR-29b could contribute to CA development.

$\mathrm{CDC7}$, a kinase involved in the initiation of DNA replication, is responsible for tumor proliferation and cell division, especially cell cycle checkpoint mechanisms $(29,30)$. For example, inhibition of CDC7 in cancer cells can induce p53-independent apoptotic cell death by impairing $\mathrm{S}$ phase progression (31). CA relates to the proliferation of VSMCs of the artery wall and possibly regulated by CDC7. In this research, we found by TargetScan that CDC7 was a credible target of miR-29b. Considering that CDC7 is overexpressed in CA tissues, it may be involved in
CA progression. The further in vitro results showed overexpression of CDC7 in HUASMCs led to promotion in proliferation, phenotypic switching, and mobility. The results further determine that CDC7 could contribute to proliferation, phenotypic switching, and mobility of HUASMCs. The luciferase reporter assay verified the direct targeting relationship between CDC7 and miR29b. Meanwhile, overexpression of miR-29b weakened the CDC7 elevation and inhibited the proliferation, phenotypic switching, and mobility of HUASMCs induced by excessive CDC7.

The interaction of miR-29b and CDC7 may also exist in other VSMC dysregulation related vascular diseases, including atherosclerosis. Though the authors found no report about $\mathrm{CDC7}$ in atherosclerosis at present, it is clear that CDC7 is a vital regulator of VSMC proliferation and phenotypic switching (12). The ability of CDC7 to promote phenotypic switching and the targeting of miR-29b strongly supported its involvement in atherosclerosis. Moreover, the role of miR-29b in atherosclerosis was indicated to be promotive of inflammation (9), and miR-29b inhibits migration and proliferation of VSMCs during neointimal formation (32). Therefore, it is also possible that targeting of miR-29b and CDC7 would benefit atherosclerosis.

There are limitations that we do not discuss other targets of miR-29b except CDC7, or more universal effects of CDC7 in CA. For instance, miR-29b was found to promote $\alpha$-SMA and SM-MHC while inhibiting MMP-2, closely related to phenotypic switching cell mobility (28). Also, HUASMC is not a satisfactory disease model to study CA, though it was used in these experiments (33). More experiments need to identify whether miR-29b targets CDC7 only in CA or targets more than one and the crosstalk between them.

In summary, our study showed miR-29b targeted CDC7 to inhibit the proliferation and mobility of HUASMCs, which may provide a potential target for CA as well as atherosclerosis.

\section{Acknowledgments}

Funding: None.

\section{Footnote}

Reporting Checklist: The authors have completed the MDAR reporting checklist. Available at http://dx.doi.org/10.21037/ atm-20-6856 
Data Sharing Statement: Available at http://dx.doi. org/10.21037/atm-20-6856

Conflicts of Interest: All authors have completed the ICMJE uniform disclosure form (available at http://dx.doi. org/10.21037/atm-20-6856). The authors have no conflicts of interest to declare.

Ethical Statement: The authors are accountable for all aspects of the work in ensuring that questions related to the accuracy or integrity of any part of the work are appropriately investigated and resolved. Written consent was obtained from each patient. The Research Ethics Committee of the Sichuan Provincial People's Hospital approved the study (No. 2018-51), according to the Declaration of Helsinki (as revised in 2013) and Title 45, US Code of Federal Regulations.

Open Access Statement: This is an Open Access article distributed in accordance with the Creative Commons Attribution-NonCommercial-NoDerivs 4.0 International License (CC BY-NC-ND 4.0), which permits the noncommercial replication and distribution of the article with the strict proviso that no changes or edits are made and the original work is properly cited (including links to both the formal publication through the relevant DOI and the license). See: https://creativecommons.org/licenses/by-nc-nd/4.0/.

\section{References}

1. Starke RM, Chalouhi N, Ding D, et al. Vascular smooth muscle cells in cerebral aneurysm pathogenesis. Transl Stroke Res 2014;5:338-46.

2. Suzuki T, Stapleton CJ, Koch MJ, et al. Decreased wall shear stress at high-pressure areas predicts the rupture point in ruptured intracranial aneurysms. J Neurosurg 2019;1:1-7.

3. Sima P, Vannucci L, Vetvicka V. Atherosclerosis as autoimmune disease. Ann Transl Med 2018;6:116.

4. Hou WZ, Chen XL, Wu W, et al. MicroRNA-370-3p inhibits human vascular smooth muscle cell proliferation via targeting KDR/AKT signaling pathway in cerebral aneurysm. Eur Rev Med Pharmacol Sci 2017;21:1080-7.

5. Li XG, Wang YB. SRPK1 gene silencing promotes vascular smooth muscle cell proliferation and vascular remodeling via inhibition of the PI3K/Akt signaling pathway in a rat model of intracranial aneurysms. CNS Neurosci Ther 2019;25:233-44.
6. Jiang $\mathrm{Y}$, Zhang $\mathrm{M}$, He $\mathrm{H}$, et al. MicroRNA/mRNA profiling and regulatory network of intracranial aneurysm. BMC Med Genomics 2013;6:36.

7. Sun L, Zhao M, Zhang J, et al. MiR-29b Downregulation Induces Phenotypic Modulation of Vascular Smooth Muscle Cells: Implication for Intracranial Aneurysm Formation and Progression to Rupture. Cell Physiol Biochem 2017;41:510-8.

8. Huang YQ, Li J, Chen JY, et al. The Association of Circulating MiR-29b and Interleukin-6 with Subclinical Atherosclerosis. Cell Physiol Biochem 2017;44:1537-44.

9. Lu Z, Wang F, Yu P, et al. Inhibition of miR-29b suppresses MAPK signaling pathway through targeting SPRY1 in atherosclerosis. Vascul Pharmacol 2018;102:29-36.

10. Cao JX, Lu Y. Targeting CDC7 improves sensitivity to chemotherapy of esophageal squamous cell carcinoma. Onco Targets Ther 2018;12:63-74.

11. Menichincheri M, Albanese C, Alli C, et al. Cdc7 kinase inhibitors: 5-heteroaryl-3-carboxamido-2-aryl pyrroles as potential antitumor agents. 1. Lead finding. J Med Chem 2010;53:7296-315.

12. Shi N, Chen SY. Cell division cycle 7 mediates transforming growth factor-beta-induced smooth muscle maturation through activation of myocardin gene transcription. J Biol Chem 2013;288:34336-42.

13. Liu D, Han L, Wu X, et al. Genome-wide microRNA changes in human intracranial aneurysms. BMC Neurol 2014;14:188.

14. Cao JX, Lu Y, Qi JJ, et al. MiR-630 inhibits proliferation by targeting CDC7 kinase, but maintains the apoptotic balance by targeting multiple modulators in human lung cancer A549 cells. Cell Death Dis 2014;5:e1426.

15. Milovic V, Teller IC, Murphy GM, et al. Deoxycholic acid stimulates migration in colon cancer cells. Eur J Gastroenterol Hepatol 2001;13:945-9.

16. Xiang SW, Xu D, Wang ZH, et al. Endovascular Treatment of Blood Blister-like Aneurysms in Carotid Atery. Journal of Sichuan University (Medical Science Edition) 2017;48:185-61.

17. Jiang $W$, Zhang $Z$, Yang $H$, et al. The miR-29b/Matrix Metalloproteinase 2 Axis Regulates Transdifferentiation and Calcification of Vascular Smooth Muscle Cells in a Calcified Environment. Blood Purif 2020;49:524-34.

18. Sun QR, Zhang X, Fang K. Phenotype of Vascular Smooth Muscle Cells (VSMCs) Is Regulated by miR-29b by Targeting Sirtuin 1. Med Sci Monit 2018;24:6599-607.

19. Bekelis K, Kerley-Hamilton JS, Teegarden A, et al. MicroRNA and gene expression changes in unruptured 


\section{Page 10 of 10}

human cerebral aneurysms. J Neurosurg 2016;125:1390-9.

20. Liu L, Liu L, Lu S. lncRNA H19 promotes viability and epithelial-mesenchymal transition of lung adenocarcinoma cells by targeting miR-29b-3p and modifying STAT3. Int J Oncol 2019;54:929-41.

21. Pan Y, Zhang Y, Liu WW, et al. LncRNA H19 overexpression induces bortezomib resistance in multiple myeloma by targeting MCL-1 via miR-29b-3p. Cell Death Dis 2019;10:106.

22. Su T, Xiao Y, Xiao Y, et al. Bone Marrow Mesenchymal Stem Cells-Derived Exosomal MiR-29b-3p Regulates Aging-Associated Insulin Resistance. ACS Nano 2019;13:2450-62.

23. Zhou S, Lei D, Bu F, et al. MicroRNA-29b-3p Targets SPARC Gene to Protect Cardiocytes against Autophagy and Apoptosis in Hypoxic-Induced H9c2 Cells. J Cardiovasc Transl Res 2019;12:358-65.

24. Qi Y, Huang Y, Pang L, et al. Prognostic value of the MicroRNA-29 family in multiple human cancers: A meta-analysis and systematic review. Clin Exp Pharmacol Physiol 2017;44:441-54.

25. Yan B, Guo Q, Fu FJ, et al. The role of miR-29b in cancer: regulation, function, and signaling. Onco Targets Ther 2015;8:539-48.

26. Liu D, Wang J, Liu M. Long noncoding RNA TUG1 promotes proliferation and inhibits apoptosis in multiple myeloma by inhibiting miR-29b-3p. Biosci Rep

Cite this article as: Ma Q, Zhang J, Zhang M, Lan H, Yang Q, Li C, Zeng L. MicroRNA-29b targeting of cell division cycle 7-related protein kinase (CDC7) regulated vascular smooth muscle cell (VSMC) proliferation and migration. Ann Transl Med 2020;8(22):1496. doi: 10.21037/atm-20-6856
Ma et al. miR-29b/CDC7 regulated VSMC proliferation and mobility

2019;39:BSR20182489.

27. Wang L, Wang Z, Huang L, et al. MiR-29b suppresses proliferation and mobility by targeting SOX12 and DNMT3b in pancreatic cancer. Anticancer Drugs 2019;30:281-8.

28. Shen L, Song Y, Fu Y, et al. MiR-29b mimics promotes cell apoptosis of smooth muscle cells via targeting on MMP-2. Cytotechnology 2018;70:351-9.

29. Kim JM, Yamada M, Masai H. Functions of mammalian Cdc7 kinase in initiation/monitoring of DNA replication and development. Mutat Res 2003;532:29-40.

30. Choschzick M, Lebeau A, Marx AH, et al. Overexpression of cell division cycle 7 homolog is associated with gene amplification frequency in breast cancer. Hum Pathol 2010;41:358-65.

31. Montagnoli A, Moll J, Colotta F. Targeting cell division cycle 7 kinase: a new approach for cancer therapy. Clin Cancer Res 2010;16:4503-8.

32. Lee J, Lim S, Song BW, et al. MicroRNA-29b inhibits migration and proliferation of vascular smooth muscle cells in neointimal formation. J Cell Biochem 2015;116:598-608.

33. Martinez AN, Pascale CL, Amenta PS, et al. Cell Culture Model to Study Cerebral Aneurysm Biology. Acta Neurochir Suppl 2020;127:29-34.

(English Language Editor: J. Chapnick) 